\title{
Coil Type Does Not Affect Angiographic Follow-Up Outcomes of Cerebral Aneurysm Coiling: A Systematic Review and Meta-Analysis
}

I. Rezek, G. Mousan, Z. Wang, M.H. Murad, and D.F. Kallmes $x={ }^{\mathrm{EBM}}$

\begin{abstract}
BACKGROUND AND PURPOSE: Previous studies varied in their conclusions about the superiority of second-generation coils compared with bare platinum. In this systematic review and meta-analysis, we assessed differences in reported unfavorable angiographic outcomes of cerebral aneurysms treated with coil embolization as a function of coil type.
\end{abstract}

MATERIALS AND METHODS: This systematic review covered 1999-2011 through the use of Ovid MEDLINE and EMBASE. Search terms were "subarachnoid hemorrhage," "intracranial aneurysms," "endovascular treatment," and "coiling." Inclusion criteria were studies reporting $>50$ aneurysms with imaging follow-up. We defined "unfavorable angiographic outcome" as either "recanalization," <90\% occlusion, or "incomplete occlusion" at follow-up. Rates of unfavorable outcomes were pooled through the use of random effects models and compared across various coil types. Multivariate random effects meta-regression models were used to further explore the differences in outcomes related to coil type.

RESULTS: We included 82 studies, comprising 90 patient cohorts, among which, 65 (72\%) used bare platinum coils, 8 (8.9\%) used Matrix, 11 (12\%) used HydroCoil, and 6 (6.7\%) used Cerecyte. The overall unfavorable outcome rate was 19\% (95\% Cl: 17\%,21\%). Unfavorable outcome rates were $20 \%$ (95\% Cl: 17\%, 22\%) for bare platinum coils, $23 \%$ (95\% Cl: 16\%, 29\%) for Matrix, $15 \%$ (95\% Cl: 9\%, $21 \%$ ) for HydroCoil, and 15\% (95\% Cl: $7 \%, 23 \%)$ for Cerecyte, respectively. The difference in unfavorable outcome rates among the various coil types was not statistically significant after adjusting for baseline characteristics, including aneurysm size, rupture status, and follow-up duration.

CONCLUSIONS: The rate of unfavorable angiographic outcomes was not statistically different across the major approved coil types. The quality of the evidence, however, remains low because of high heterogeneity, small sample size, and potential publication bias.

ABBREVIATIONS: MeSH = Medical Subject Headings; $\mathrm{RCT}=$ randomized, controlled trial; IQR $=$ interquartile range

D

espite widespread acceptance of endovascular coil embolization for aneurysms, rates of unfavorable angiographic outcomes remain relatively high. A recent systematic review of coiling literature reported a rate of $18 \%$ of such outcomes. ${ }^{1}$ In an effort to improve long-term occlusion rates, a number of "modified," second-generation coil types have been developed and marketed. Numerous single-center case series have been published regarding outcomes for each of these second-generation coil types, including Matrix (Boston Scientific, Natick, Massachu-

Received December 12, 2012; accepted after revision March 13, 2013.

From the Department of Radiology (I.R., D.F.K.), The Center for the Science of Health Care Delivery (Z.W., M.H.M.), and Department of Neurosurgery (D.F.K.), Mayo Clinic, Rochester, Minnesota; and Children's Hospital of Michigan (G.M.) Detroit, Michigan.

Please address correspondence to Issa Rezek, MD, Department of Radiology, Mayo Clinic, 200 1st St SW, Rochester, MN 55905; e-mail: rezek.issa@mayo.edu

Indicates article with supplemental on-line appendix.

Evidence-Based Medicine Level 1.

http://dx.doi.org/10.3174/ajnr.A3598 setts), ${ }^{2-4}$ HydroCoil (MicroVention, Tustin, California), ${ }^{5-7}$ and Cerecyte coils (Codman Neurovascular, Raynham, Massachusetts). ${ }^{8,9}$ In addition, outcomes from several randomized, controlled trials (RCTs) of the major coil types have recently been reported. ${ }^{10-12}$

To date, relatively few literature reviews of second-generation coils have been published, and such reviews rarely if ever have implemented formal meta-analytic procedures. ${ }^{13,14}$ In this current study, we report results from a systematic review and metaanalysis assessing differences in reported unfavorable angiographic outcomes for cerebral aneurysms treated with coil embolization as a function of coil type.

\section{MATERIALS AND METHODS}

The methodology and reporting of this systematic review follows the Preferred Reporting Items for Systematic Reviews and Metaanalyses (PRISMA) statement. ${ }^{15}$

\section{Literature Search}

We searched Ovid MEDLINE and EMBASE databases from January 1999 to December 2011. The search was performed by an 
experienced reference librarian. The following key words as Medical Subject Headings (MeSH) terms and text words were used in relevant combinations: "subarachnoid hemorrhage," "intracranial aneurysm," "endovascular treatment," and "coiling" in both "AND" and "OR" combinations. In addition, we reviewed abstracts from selected radiology, neurology, and neurosurgery scientific meetings in the years 2011 and 2012 to identify coil studies (the 2012 annual meeting of the American Society of Neuroradiology, the 2011 annual meeting of the Society of Neurointerventional Surgery, and the 2011 annual meeting of the Congress of Neurologic Surgeons).

Inclusion criteria were 1) >50 aneurysms reported, 2) coil type clearly specified and noted to be either bare platinum, Matrix, HydroCoils, or Cerecyte coils, and 3) available imaging follow-up with DSA or MR angiography. If both MRA and DSA outcomes were available, we included DSA results. If DSA was not available, MR angiography was then used.

Exclusion criteria were 1) traumatic, dissecting, mycotic, or flowrelated aneurysms, 2) treatment with other coil types or lack of clarity regarding coil type used, primarily stent-treated aneurysms, or noncoil embolic agents used to perform either aneurysm or parent vessel coil occlusions. When studies had multiple groups comparing different imaging modalities (eg, DSA, MRA), only the groups that were followed with DSA were considered. When the same patient population was the subject of multiple publications, only the study with the largest cohort was included in this analysis.

The primary outcome was "unfavorable" angiographic outcome, defined as any degree of recanalization noted on the follow-up images with comparison to the immediate posttreatment results. Terms such as "aneurysm recurrence," "new filling of aneurysm lumen," and "regrowth" were considered synonymous with "recanalization." If "recanalization" was not reported in a study, then the "unfavorable angiographic outcome" was defined as either $<90 \%$ degree of occlusion or class 3 on the Raymond scale (incomplete occlusion), which is defined as any opacification of the sac. ${ }^{16}$ We considered the longest duration of reported angiographic results for each study when more than 1 phase of follow-up was reported.

Each of 2 reviewers (I.R., G.M.) independently evaluated the articles in the librarian's primary list and selected studies that fulfilled the inclusion criteria. In the case of a disagreement, D.F.K. reviewed and adjudicated inclusion. For each study, we extracted number of coiled aneurysms, number of aneurysms that had available follow-up, mean aneurysm size, initial rupture status, mean duration of follow-up, use of a core laboratory facility, whether assessed by single or multiple readers, angiographic follow-up outcomes, and coil types.

In this present review, we classified 4 types of coils: bare platinum, Matrix, HydroCoil, and Cerecyte. Guglielmi detachable coils were considered to be equivalent to bare platinum coils. Matrix coils included all Matrix subtypes (Matrix 1, Matrix 2, Matrix ACTIVE, and Matrix POST-ACTIVE). The HydroCoil group included studies that used either HydroCoil or HydroSoft. Cerecyte studies were considered as Cerecyte when their use was clearly stated in the methods.

\section{Statistical Analysis}

We used the analysis of variance test to compare baseline group characteristics (mean aneurysm size, proportion of initially rup-

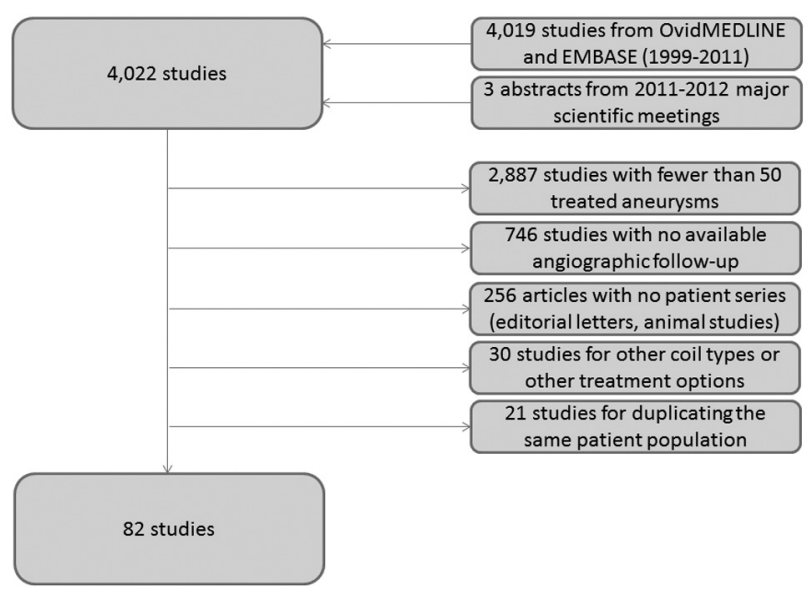

FIG 1. Search strategy in a flow chart along with reasons for exclusion.

tured aneurysms, and mean follow-up duration). We calculated the rates of unfavorable angiographic outcomes from each study. The confidence intervals of the rates were estimated by the Jeffreys method. ${ }^{17}$ We used the DerSimonian and Laird random effects method to pool the overall rate of unfavorable outcome as well as the rate of unfavorable outcome for each coil type. ${ }^{18,19}$ To compare rates of unfavorable outcomes among coil types, we used the test of interaction proposed by Altman and Bland. ${ }^{20}$ We also constructed multivariate nested random effects meta-regression models to further explore the difference of coil types after adjusting for baseline characteristics. ${ }^{19}$

We used the $I^{2}$ statistic and the Cochran $Q$ test to measure the overall heterogeneity, which refers to the variations of study outcomes across the studies. $I^{2}>50 \%$ and a conservative $P$ value $(P<$ .10) suggest high heterogeneity. ${ }^{21}$ The Tau ${ }^{2}$ statistic was adopted to measure the variance across studies. We also used the $R^{2}$ statistic to measure the proportion of total variance explained by each coil type. With the use of the Egger regression asymmetry test, we also assessed whether our findings were likely to be biased by the tendency that significant results are more likely to be published, so-called publication bias. ${ }^{22}$ All statistical analyses were conducted with the use of STATA version 12 (StataCorp, College Station, Texas). We used the GRADE framework to evaluate the overall quality of the evidence (ie, confidence in the estimates). ${ }^{23,24}$

\section{Study Quality}

The quality of the included studies was assessed by use of the New castle-Ottawa Quality Assessment Scale. ${ }^{25}$ We selected the scale items relevant to uncontrolled studies, such as most the included studies, and therefore quality evaluation focused on the following items: representativeness of the exposed cohort to the population at hand (truly or somewhat representative versus selected groups or no description), selection of the nonexposed cohort (drawn from the same population versus not or no description), ascertainment of exposure (coiling procedure recorded), assessment of the outcome (independent versus self-report), length of follow-up ( $>6$ months or $<6$ months), and loss to follow-up ( $>75 \%$ of the studied aneurysms were followed versus less). 
Table 1: Baseline characteristics of study groups

\begin{tabular}{lcccc}
\hline Coil Group & $\begin{array}{c}\text { Number of Studied } \\
\text { Groups (\%) }\end{array}$ & $\begin{array}{c}\text { Mean Aneurysm } \\
\text { Size, mm (SD) }\end{array}$ & $\begin{array}{c}\text { Percentage of Initially } \\
\text { Ruptured Aneurysms (SD) }\end{array}$ & $\begin{array}{c}\text { Mean Follow-Up } \\
\text { Duration, mo (SD) }\end{array}$ \\
\hline Total & $90(10)$ & $8.0(3.1)$ & $64(28)$ & $18.9(19.8)$ \\
Bare platinum & $65(72)$ & $8.2(3.5)$ & $66(30)$ & $22.2(22.2)$ \\
Matrix & $8(8.9)$ & $7.5(1.8)$ & $55(16)$ & $9.4(3.2)$ \\
HydroCoil & $11(12)$ & $7.9(1.2)$ & $58(23)$ & $10.3(4.2)$ \\
Cerecyte & $6(6.7)$ & $6.3(7.9)$ & $72(22)$ & $11.2(9.0)$ \\
\hline
\end{tabular}

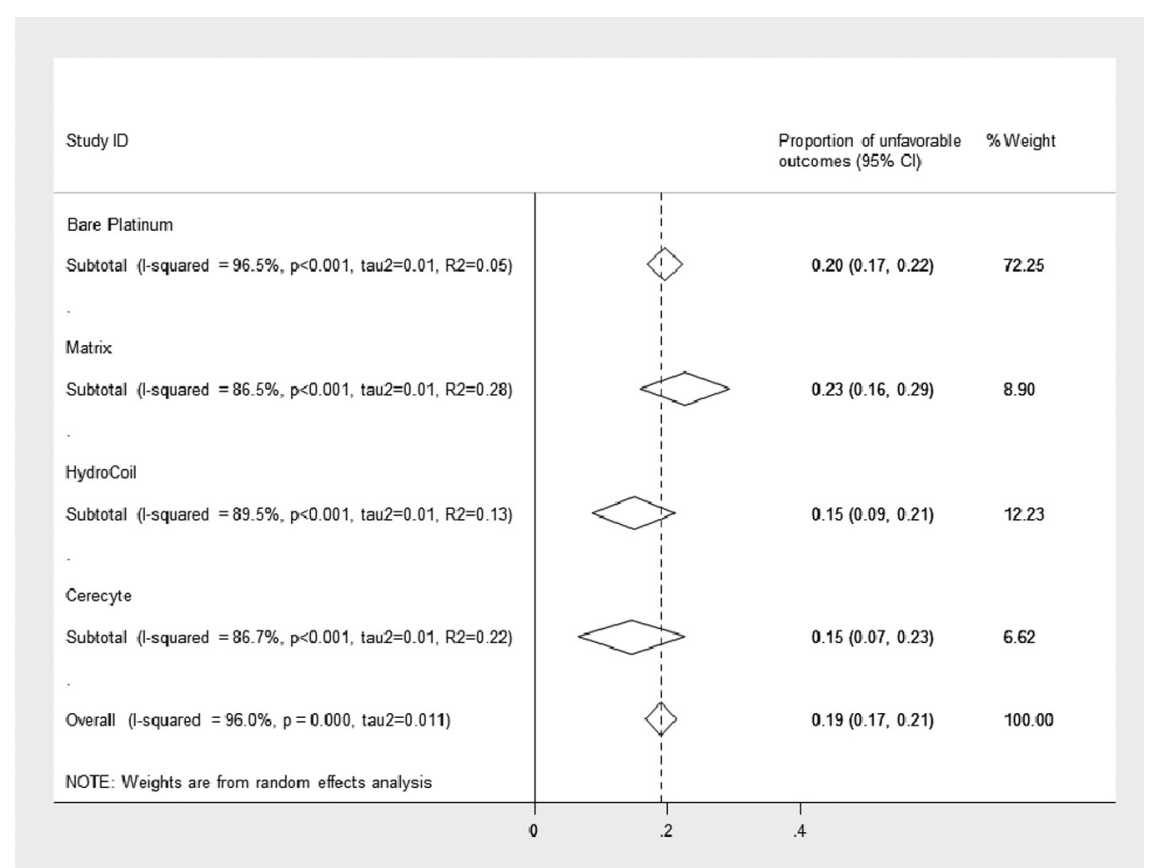

FIG 2. Forest plot shows the pooled outcome estimates from random effects analysis.

The average percentage of ruptured aneurysms in the enrolled studies was $64 \%$ (IQR: $11-100 \%)$. The patients were followed up to 18.9 months (IQR: 4.5-69 months) on average.

Sixty-five $(72 \%)$ of 90 patient subgroups were treated with bare platinum coils, 8 subgroups $(8.9 \%)$ were treated with Matrix coils, 11 (12\%) were treated with HydroCoils, and 6 (6.7\%) were treated with Cerecyte. Table 1 summarizes baseline characteristics of the studied groups. The differences among coil types were not significant regarding mean aneurysm size $(P=.69)$, the proportion of initially ruptured aneurysms $(P=.58)$, or the mean duration of follow-up $(P=.08)$.

The pooled rates of unfavorable outcomes are shown in Fig 2. Overall, the rate of unfavorable outcomes from all studies was 19\% (95\% CI: 17\%, 21\%) (2452 of 12,986 aneurysms). For bare platinum coils, the rate was $20 \%(95 \%$

\section{RESULTS}

We identified 4019 potential references published between January 1999 and December 2011 as well as 3 prospective coil studies identified from 2011-2012 conference proceedings ${ }^{11,12,26}$ (Fig 1). There was a disagreement on 8 studies $(0.2 \%)$ between the 2 reviewers that was resolved by a decision from the senior author. A total of 3940 studies (97\%) of 4022 were excluded for the following reasons: $<50$ treated aneurysms ( 2887 articles; $72 \%$ ), no available angiographic follow-up (746 articles; 19\%), nonclinical series (editorial letters, nonhuman experimental models for cerebral aneurysms) (256 articles; $6 \%$ ), utilization of coil types other than the designated included categories or treatment options other than coiling, such as stents alone or parent artery occlusion (30 articles; $0.8 \%$ ), and duplicate publishing of the same patient population (21 articles; 0.5\%). As such, 82 (2\%) studies met all inclusion criteria and were included in our analysis (see On-line Appendix for full list of included studies). Seventy-four (90\%) of the 82 studies described patients treated with a single coil type. The remaining 8 studies of the included 82 (10\%) compared 2 subsets of patient groups that were treated with 2 different coil types. Each subset was considered a separate patient subgroup. In total, there were 90 patient subgroups.

The total number of treated aneurysms was 17,706, of which $12,986(73 \%)$ had available angiographic follow-up outcomes and were included in the final analysis. The overall mean aneurysm size was $8.0 \mathrm{~mm}$ (interquartile range [IQR]: 4.6-15 mm).
CI: $17 \%, 22 \%$ ) (1907 of 10,370 aneurysms), for Matrix it was $23 \%$ (95\% CI: 16\%, 29\%) (279 of 1128 aneurysms), for HydroCoil it was $15 \%$ (95\% CI: 8.7\%, 21\%) (175 of 977 aneurysms), and for Cerectye it was $15 \%$ (95\% CI: 6.6\%, 23\%) (91 of 511 aneurysms). The difference in rates across various coil types was not statistically significant $(P=.32)$. Even after adjusting for baseline rupture, aneurysm size, follow-up time, core laboratory, and multiple-reader interpretation effect, we found no significant differences in the reported unfavorable outcomes rates between coil types (Table 2).

Across studies, substantial heterogeneity was observed in all pooled outcome estimates $\left(I^{2}>50 \%, P<.001\right)$ (Fig 2). The Egger regression asymmetry test suggested potential publication bias $(P<.001)$. The quality of the included studies is summarized in Fig 3. Only 3 (3.7\%) of 82 studies were RCTs. ${ }^{10,12}$ The remaining studies were observational cohort studies with or without a control group. Fig 3 summarizes the quality of the included studies. Selection of nonexposed cohorts was not adequate because most of the studies described a single-center series of patients with no control group. The assessment of outcome was self-reported except for $8(9.8 \%)$ of 82 studies in which an independent core laboratory reported the angiographic outcomes. The other items were found to be adequate in the included studies. The overall quality of the current evidence (ie, confidence in the estimates) is 
low, considering the observational nature of most of the studies, heterogeneity, and the likelihood of publication bias.

\section{DISCUSSION}

In the current study, with the use of formal meta-analytic tools, we found that reported rates of unfavorable angiographic outcomes were not significantly different among the various leading types of endovascular coils for aneurysm therapy. Overall, approximately 1 in 5 aneurysms showed unfavorable outcome at follow-up. At first glance, both HydroCoil and Cerecyte rates appear to be superior to bare platinum and Matrix, with the former coil types showing $15 \%$ and the latter coil types showing $20 \%$ unfavorable outcome rates. However, given the relatively small numbers of aneurysms, even when pooling studies, the confidence intervals for all reported proportions were wide and thus significant differences were not shown. The results from this current study were further compromised by substantial heterogeneity among studies as well as by signals of potential publication bias. Taken together, these results suggest that clear differences among coil type have yet to be manifest and that implementation of future, high-quality, prospective studies remains of paramount importance.

Previous studies have failed to demonstrate consensus regarding the relative advantages of second-generation coils compared with bare platinum coils. Whereas some previous studies were encouraging for improved outcomes, ${ }^{7,27-30}$ others were not as promising. ${ }^{13,31,32}$ Three recent RCTs compared

Table 2: Multivariate random effects meta-regression

\begin{tabular}{lccc}
\hline \multicolumn{1}{c}{ Variable } & Odds Ratio & $\begin{array}{c}\text { 95\% Confidence } \\
\text { Interval }\end{array}$ & $\boldsymbol{P}$ Value \\
\hline Bare platinum & Reference & & \\
Matrix & 0.93 & $0.30,2.89$ & .90 \\
HydroCoil & 0.46 & $0.17,1.25$ & .13 \\
Cerecyte & 0.58 & $0.17,1.99$ & .38 \\
\hline
\end{tabular}

bare platinum coils with each of the leading modified coils, including HydroCoil, ${ }^{10}$ Matrix, ${ }^{11}$ and Cerecyte coils. ${ }^{12}$ The HELPS trial found a slight improvement in major recurrences among aneurysms that were treated with HydroCoils. However, the MAPS and Cerecyte trials found no significant advantages of newer devices over bare platinum.

Previous reviews of the literature have assessed possible advantages of Matrix coils ${ }^{13}$ and other second-generation coils. ${ }^{14}$ The results of our current study are relatively consistent with previous studies, in that none has found significant differences between coil types on angiographic follow-up. However, our current study improves on this prior literature in several ways. First, previous studies comprised studies up to the year $2005^{13}$ or $2007^{14}$; therefore, our results bring the evidence base up to date. Furthermore, with the use of formal meta-analytic techniques, we accounted for potential confounding variables such as aneurysm size, baseline rupture status, and duration of follow-up, all of which can impact rates of unfavorable angiographic outcome. Finally, our data point to substantial heterogeneity as well as potential publication bias, which mandate that all of these results should undergo careful scrutiny by practitioners.

Our study has many limitations. The heterogeneity of the studies in the literature forced us to combine patients that were followed by either DSA or MR angiography despite possible technique-related variations and combine multiple types of unfavorable results, including incomplete occlusion and Raymond class 3 , with recanalization. Also, most studies were excluded for incomplete reporting of outcomes. We did not consider other variables that would potentially affect outcomes, including proportion of wide-necked aneurysms and anatomic location. The lack of statistical significance may also be due underpowered subgroup analyses and should not be interpreted as equivalence of the different coil types. Finally, in this systematic review, we found only 3 RCTs. Observational studies are subject to high risk of bias be-

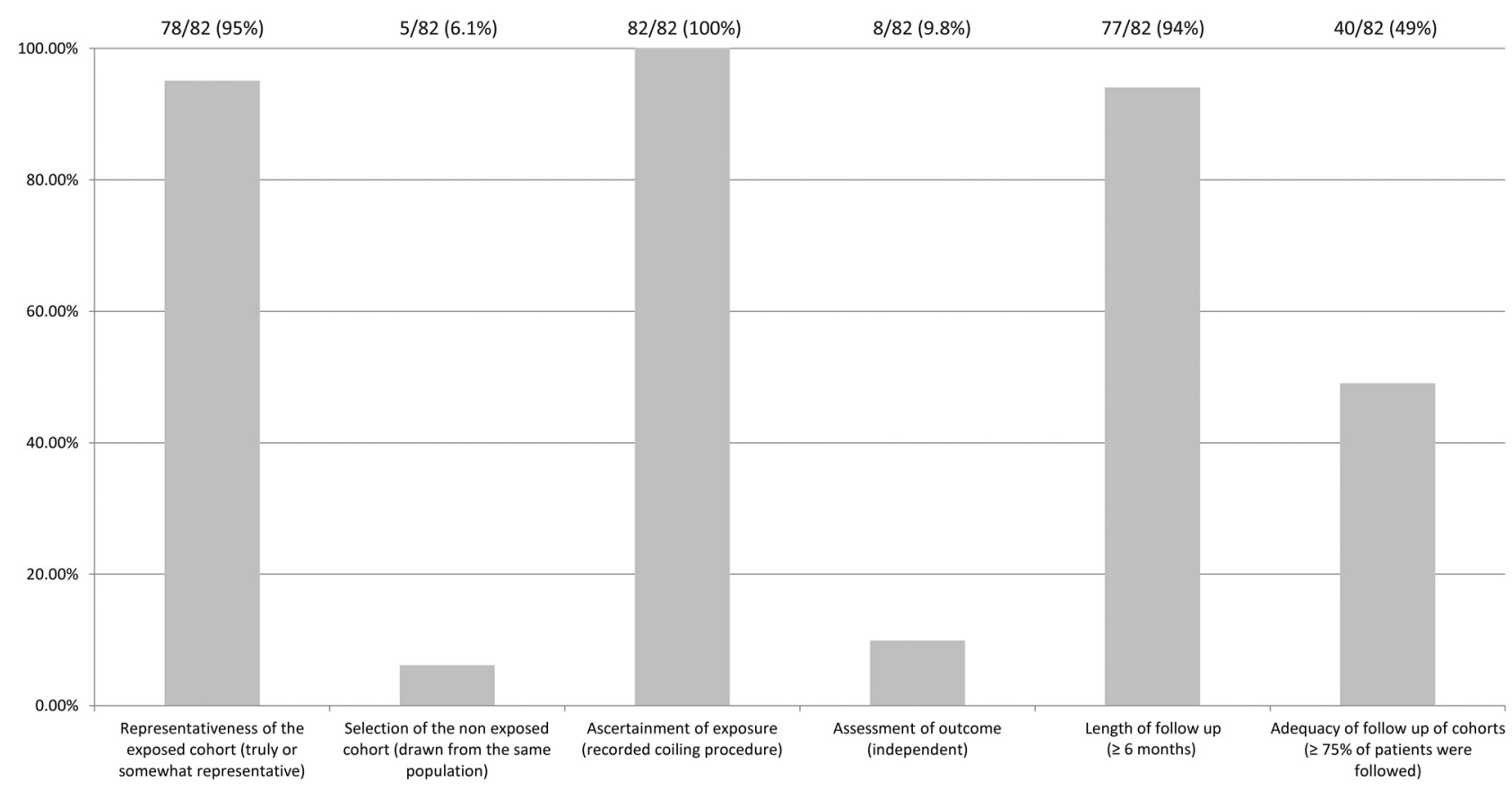

FIG 3. Quality assessment of the included studies. 
cause of baseline imbalance and potential outcome confounding. By pooling results from observational studies and RCTs, ecological bias may have affected our results. Further studies are clearly needed to provide higher quality evidence.

\section{CONCLUSIONS}

The rate of unfavorable angiographic outcomes of cerebral aneurysms treated with coil embolization was not statistically different across the major approved coil types.

Disclosures: David F. Kallmes—UNRELATED: Consultancy: ev3,* Codman,* Medtronic*; Grants/Grants Pending: ev3,* MicroVention, ${ }^{\star}$ Sequent, ${ }^{\star}$ Codman*; Payment for Lectures (including service on Speakers Bureaus): MicroVention*; Royalties: UVA Patent Foundation*; payment for development of educational presentations: ev3*; Travel/Accommodations/Meeting Expenses unrelated to activities listed: MicroVention* ( ${ }^{*}$ money paid to institution).

\section{REFERENCES}

1. Rezek I, Mousan G, Wang Z, et al. Effect of core laboratory and multiple-reader interpretation of angiographic images on follow-up outcomes of coiled cerebral aneurysms: a systematic review and meta-analysis. AJNR Am J Neuroradiol 2013;34:1380-84

2. Linfante I, Akkawi NM, Perlow A, et al. Polyglycolide/polylactide-coated platinum coils for patients with ruptured and unruptured cerebral aneurysms: a single-center experience. Stroke 2005;36:1948-53

3. Mitra D, Herwadkar A, Soh C, et al. Follow-up of intracranial aneurysms treated with Matrix detachable coils: a single-center experience. AJNR Am J Neuroradiol 2007;28:362-67

4. Ishii A, Murayama Y, Nien YL, et al. Immediate and midterm outcomes of patients with cerebral aneurysms treated with Matrix1 and Matrix 2 coils: a comparative analysis based on a single-center experience in 250 consecutive cases. Neurosurgery 2008;63:1071-79

5. Berenstein A, Song JK, Niimi Y, et al. Treatment of cerebral aneurysms with hydrogel-coated platinum coils (HydroCoil): early single-center experience. AJNR Am J Neuroradiol 2006;27:1834-40

6. Deshaies EM, Adamo MA, Boulos AS. A prospective single-center analysis of the safety and efficacy of the Hydrocoil embolization system for the treatment of intracranial aneurysms. J Neurosurg 2007; 106:226-33

7. Gunnarsson T, Tong FC, Klurfan P, et al. Angiographic and clinical outcomes in 200 consecutive patients with cerebral aneurysm treated with hydrogel-coated coils. AJNR Am J Neuroradiol 2009;30:1657-64

8. Butteriss D, Gholkar A, Mitra D, et al. Single-center experience of Cerecyte coils in the treatment of intracranial aneurysms: initial experience and early follow-up results. AJNR Am J Neuroradiol 2008;29:53-56

9. Geyik S, Yavuz K, Ergun O, et al. Endovascular treatment of intracranial aneurysms with bioactive Cerecyte coils: effects on treatment stability. Neuroradiology 2008;50:787-93

10. White PM, Lewis SC, Gholkar A, et al. Hydrogel-coated coils versus bare platinum coils for the endovascular treatment of intracranial aneurysms (HELPS): a randomized controlled trial. Lancet 2011;377:1655-62

11. Claiborne Johnson S, McDougal C, Gholkar A. The MAPS (Matrix and Platinum Science) Trial: primary results. In: Proceedings of the Eighth Annual Meeting of the Society of Neurointerventional Surgery, Colorado Springs, Colorado: July 25-28, 2011

12. Molyneaux AJ, Fox A, Sneade M, et al. Cerecyte Coil Trial: angiographic and clinical outcomes of endovascular coiling in patients with ruptured and unruptured intracranial aneurysms treated with Cerecyte coils compared with bare platinum coils: finals results of a prospective randomized trial. In: Proceedings of the American Society of Neuroradiology 50th Annual Meeting and the Foundation of the ASNR Symposium, New York: April 21-26, 2012

13. Smith MJ, Mascitelli J, Santillan A, et al. Bare platinum vs Matrix detachable coils for the endovascular treatment of intracranial aneurysms: a multivariate logistic regression analysis and review of the literature. Neurosurgery 2011;69:557-65

14. White PM, Raymond J. Endovascular coiling of cerebral aneurysms using "bioactive" or coated-coil technologies: a systematic review of the literature. AJNR Am J Neuroradiol 2009;30:219-26

15. Moher D, Liberati A, Tetzlaff J, et al. Preferred reporting items for systematic reviews and meta-analyses: the PRISMA statement. Ann Intern Med 2009;151:264-69

16. Roy D, Milot G, Raymond J. Endovascular treatment of unruptured aneurysms. Stroke 2001;32:1998-2004

17. Clopper C, Pearson ES. The use of confidence or fiducial limits illustrated in the case of the binomial. Biometrika 1934;26:404-13

18. DerSimonian R, Laird N. Meta-analysis in clinical trials. Controlled Clin Trials 1986;7:177-88

19. Freemantle N, Cleland J, Young P, et al. Beta blockade after myocardial infarction: systematic review and meta regression analysis. BMJ 1999;318:1730-37

20. Altman DG, Bland JM. Interaction revisited: the difference between two estimates. BMJ 2003;326:219

21. Julian PTH, Simon GT, et al. Measuring inconsistency in meta-analyses. BMJ 2003;327:557-60

22. Egger M, Davey Smith G, Schneider M, et al. Bias in meta-analysis detected by a simple, graphical test. BMJ 1997;315:629-34

23. Balshem $H$, Helfand $M$, Schunemann HJ, et al. GRADE guidelines: 3 , rating the quality of evidence. J Clin Epidemiol 2011;64:401-06

24. Guyatt GH, Oxman AD, Vist G, et al. GRADE guidelines: 4, rating the quality of evidence-study limitations (risk of bias). J Clin Epidemiol 2011;64:407-15

25. Wells GA, Shea B, O'Connell D, et al. The Newcastle-Ottawa Scale (NOS) for assessing the quality of nonrandomised studies in metaanalyses. Third Symposium on Systematic Reviews: Beyond the Basics. 2000 July; Oxford, UK.

26. Pierot L, Cognard C, Ricolfi F, et al. Mid-term anatomic results after endovascular treatment of ruptured intracranial aneurysms with GDC and Matrix coils: analysis of the CLARITY series. AJNR Am J Neuroradiol 2012;33:469-73

27. Murayama Y, Viñuela F, Ishii A, et al. Initial clinical experience with Matrix detachable coils for the treatment of intracranial aneurysms. J Neurosurg 2006;105:192-99

28. Park JH, Kang HS, Han MH, et al. Embolization of intracranial aneurysms with HydroSoft coils: results of the Korean multicenter study. AJNR Am J Neuroradiol 2011;32:1756-61

29. Cloft HJ, Kallmes DF. Aneurysm packing with HydroCoil embolic system versus platinum coils: initial clinical experience. AJNR Am J Neuroradiol 2004;25:60-62

30. O'Hare AM, Fanning NF, Ti JP, et al. HydroCoils, occlusion rates, and outcomes: a large single-center study. AJNR Am J Neuroradiol 2010;31:1917-22

31. Roth C, Struffert T, Grunwald IQ, et al. Long-term results with Matrix coils vs GDC: an angiographic and histopathological comparison. Neuroradiology 2008;50:693-99

32. Piotin M, Spelle L, Mounayer C, et al. Intracranial aneurysms coiling with Matrix: immediate results in 152 patients and midterm anatomic follow-up from 115 patients. Stroke 2009;40:321-23 